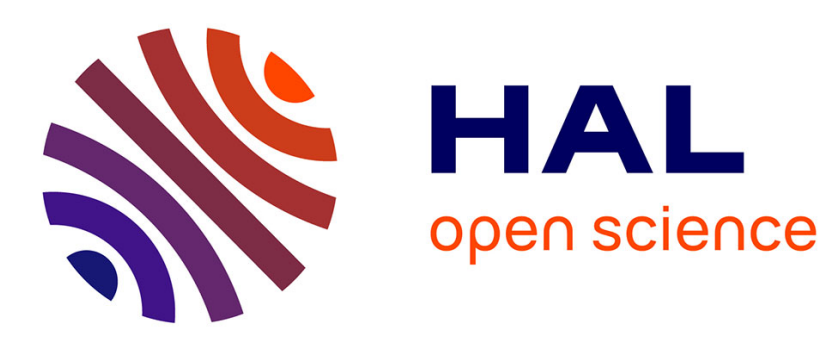

\title{
Moustapha Safouan: La psychanalyse. Science, thérapie - et cause
}

\author{
Christian Hoffmann
}

\section{To cite this version:}

Christian Hoffmann. Moustapha Safouan : La psychanalyse. Science, thérapie - et cause. Figures de la psychanalyse, 2014, L'Hystérie, 1 (27 ), pp.193 - 198. 10.3917/fp.027.0193 hal-01511872

\section{HAL Id: hal-01511872 \\ https://hal.science/hal-01511872}

Submitted on 25 Aug 2017

HAL is a multi-disciplinary open access archive for the deposit and dissemination of scientific research documents, whether they are published or not. The documents may come from teaching and research institutions in France or abroad, or from public or private research centers.
L'archive ouverte pluridisciplinaire HAL, est destinée au dépôt et à la diffusion de documents scientifiques de niveau recherche, publiés ou non, émanant des établissements d'enseignement et de recherche français ou étrangers, des laboratoires publics ou privés. 


\title{
Moustapha Safouan : La psychanalyse. Science, thérapie - et cause 1
}

\author{
- Christian Hoffmann •
}

Psychanalyste, avec soixante ans d'expérience de la cure et du mouvement psychanalytique, c'est de cette position singulière que Moustapha Safouan fait œuvre dans ce livre. Il ne nous transmet pas une Summa, dans laquelle le lecteur risquerait de se perdre ou de se sentir écrasé. Tout au contraire, dans la première partie consacrée au « mouvement freudien ", Moustapha Safouan analyse ce mouvement qui a abouti à la création de l'Association psychanalytique internationale dont le but était de veiller par la formation des analystes aux risques de déviations, tant à l'œuvre qu'à la personne de Freud. Le lecteur peut rapidement attraper le fil rouge qui le guidera à travers ce livre, il suffit pour cette saisie de comprendre que Moustapha Safouan va lire les drames et les ruptures dans le mouvement freudien en ne cédant pas sur l'analyse au profit de la doctrine et de son organisation, et ceci jusqu'à Freud lui-même. Dans la seconde partie sur " La théorie psychanalytique de l'Éros ", le lecteur est invité à redécouvrir que le génie de Freud se trouve tout particulièrement dans son invention de la pulsion. C'est avec ce concept de pulsion que la psychanalyse peut développer un savoir « scientifique de l'Éros ", en levant le voile sur son apparition précoce dans la subjectivité et sur son objet. Un objet perdu qui ne trouve son sens qu'à partir de la lecture de la « phase phallique » comme introduisant un tiers terme qui est le phallus (qu'on n'est pas), par lequel Lacan réinvente la psychanalyse en lui donnant une autre perspective que celle de l'avoir ou pas. La révision par Lacan de l'OEdipe centre le complexe sur la valeur phallique (la castration) qui s'accroche à d'autres objets que le pénis. Il en résulte la sexualisation des objets. C'est avec cette clef que Moustapha Safouan nous ouvre la porte de la logique de la sexuation dans la théorie lacanienne. Dans la troisième partie sur « La saga lacanienne », l'orientation nous est donnée par le désir de Lacan de développer une pratique qui soit à la hauteur scientifique de la psychanalyse, jusque dans sa transmissibilité. Ce qui nous permet de comprendre

1. Moustapha Safouan, La psychanalyse. Science, thérapie - et cause, Vincennes, éditions Thierry Marchaisse, 2013. 
la déception de Lacan quant aux résultats attendus de « la passe », dont la procédure visait à la production d'un savoir sur « le désir qui fait l'analyste ». L'implication permanente de Moustapha Safouan dans le jury de la passe donne tout son poids à son analyse de son « échec », à savoir que cet échec est celui de l'attendu d'un savoir sur un désir, ce qui s'est soldé par la preuve de l'impossible. Moustapha Safouan n'hésite pas à nous donner son expérience personnelle de son lien à Lacan, ce qui lui permet de distinguer le maître avec qui il travaillait comme « élève » et celui de son École et de son séminaire. La conclusion de Moustapha Safouan va au cœur du mouvement psychanalytique sur l'antinomie entre la psychanalyse et l'institutionnalisation de sa cause. Rank en pointait déjà « la fiction », ce qui nous incite après la présentation du fil rouge de ce livre à en développer l'argumentation.

Dès les premières pages de la première partie sur le « Mouvement freudien », et ceci depuis la «Société du mercredi », voulue par Stekel, à la création du « comité secret » sous l'impulsion de Jones, Moustapha Safouan pose la question qui interroge ce « mouvement » au regard de la science de son époque : «La question est de savoir comment et pourquoi un homme de science tel que lui (Freud) en est arrivé à se mettre dans la position d'un chef de mouvement ». Au congrès de Nuremberg, Ferenczi a nommé le groupe formé par les premiers psychanalystes « communauté familiale ». Les aspects pathologiques de ces communautés ne lui ont pas échappé et il a proposé que « Des membres ayant reçu une formation psychanalytique seraient donc à même de fonder une association réunissant les avantages de l'organisation familiale à un maximum de liberté individuelle ». On appréciera la justesse de l'analyse de
Moustapha Safouan sur le retrait de Freud de cette organisation internationale au profit de Jung, un retrait qui ne souligne que plus sa position de «père » d'une nouvelle science réunissant une large famille qui nécessite la désignation d'un « prince héritier ». Son argumentation nous amène aux deux piliers de la création de l'IPA, qui sont pour lui indéfendables, la structure familiale de son organisation pour défendre « la cause » et la résistance à « l'intérêt commun » et aux « thèses centrales » que seule l'analyse serait à même de vaincre.

Il n'est par conséquent pas étonnant de découvrir que l'institutionnalisation de la formation des analystes cherche à garantir cette formation pour remédier aux dangers internes de déviations des analystes. L'enseignement de la psychanalyse et l'analyse de contrôle sont apparus avec ce besoin de garantie. Le prix à payer est celui d'un appauvrissement de la recherche au profit du dogme. Cette transmission institutionnelle de la psychanalyse s'est vite soldée par un échec, la démission de Jung et la création du « comité secret » sous l'impulsion de Jones, qui y voyait un lieu possible pour y exercer son pouvoir. Dans ce « comité secret » siégeaient les deux « cardinaux », Jones et Abraham, et les deux " hérétiques », Rank et Ferenczi. Eitingon et Sachs sont venus plus tard.

Le chapitre sur $O$. Rank est non seulement un argument de taille pour la thèse de Moustapha Safouan sur la disjonction entre l'analyse et son organisation, mais il témoigne aussi du déchiffrage analytique de la surdité de Freud au transfert et au travail de transfert de Rank. Le lecteur découvrira l'hypothèse analytique de Moustapha Safouan concernant Le traumatisme de la naissance, tant sur sa place dans ce transfert 
que sur son apport d'une nouveauté concernant le rapport de la castration à l'CEdipe et au désir, qui avoisine Lacan. Le lecteur pourra découvrir que Rank fut, avec Ferenczi, le seul analyste innovant dans ce mouvement.

Le chapitre suivant traite tout naturellement deS. Ferenczi, qui, avec Rank, avait un «transfert fécond sur Freud ». Il a payé cher ce transfert, pour des raisons similaires à Rank, du fait que Freud a choisi dans ce transfert de servir la cause de la psychanalyse plutôt que celle de la vérité de la parole. Là aussi, le lecteur lira l'analyse par Moustapha Safouan de la position de Freud et ses conséquences sur celui qu'il appelait son « fils », qu'il a poussé dans les bras d'une femme qui le coupait de la paternité. Les apports de Ferenczi à la psychanalyse sont considérables. Sa contribution à la question de la fin de l'analyse est essentielle par la mise en lumière de la relation entre le mensonge et la jouissance du fantasme et sa renonciation qui constitue un progrès considérable dans la cure. Ce que Lacan appellera la traversée du fantasme. L'hostilité marquée de Freud à l'exposé sur « La confusion des langues » a précipité la rupture entre les deux hommes. Son hypothèse sur la dépendance de la sexualité de l'enfant à celle de l'adulte, qui nous permet avec Lacan de comprendre le désir comme désir de l'Autre, était aux antipodes de ce qu'on pouvait supporter d'entendre à l'époque. Avec le rappel de la plus grande difficulté de l'analyse que Ferenczi reconnaissait dans «la démolition de l'image paternelle », Moustapha Safouan peut traduire l'apport de Ferenczi par la découverte anticipée de « la transformation lacanienne de l'CEdipe en fonction phallique ». Le père de la psychanalyse l'a entendu d'une autre oreille et nous connaissons la suite du destin de Ferenczi.
Cette première partie sur « Le mouvement freudien », qui donne une large place à l'ouvrage de Rank et de Ferenczi Perspectives de la psychanalyse, nous transporte dans une « Église » avec ses « cardinaux » qui ont enterré les deux « hérétiques », ceux qui ont osé mettre en question les dogmes de l'analyse didactique et de son caractère enseignant. Le résultat que Moustapha Safouan y voit, c'est qu'on n'a pas avancé d'un pouce jusqu'à aujourd'hui sur le passage à l'analyste et sur la fonction du didacticien. On appréciera la discussion des innovations de nos deux hérétiques sur la répétition, l'interprétation et la guérison par le savoir.

Le lecteur qui a suivi attentivement le débat antérieur sur l'@Edipe et la castration, qui anticipe la théorie du désir de Lacan, se retrouvera dans la seconde partie du livre où il pourra se familiariser avec « La théorie psychanalytique de l'Éros».

Moustapha Safouan a rencontré Lacan en 1949, il commente le retour à Freud de Lacan auquel il a participé, comme un travail de conceptualisation rigoureuse et scientifique de Freud dont les effets étaient attendus dans la cure. Cette « rénovation conceptuelle » a très vite abandonné « la guérison par le savoir » en reconnaissant la disjonction entre le savoir de l'analyste et la vérité du patient, qui elle-même se distingue de son savoir. La boucle avec Rank et Ferenczi est ainsi faite. Le travail de Lacan a fait basculer l'interrogation de la relation patient-analyste vers l'étude de la relation du sujet à sa parole, du fait même que le désir détermine le sujet dans ses actes comme dans sa parole. La linguistique est venue se substituer à la référence biologique. C'est ainsi que Moustapha Safouan renouvelle la question de la différence des sexes en s'ap- 
puyant sur la conception saussurienne d'un langage qui n'est que différence. La subordination de l'être au langage introduit une rupture ontologique où la question de la définition, par exemple de ce qu'est la femme, ne peut se résoudre qu'à l'intérieur de ce système. Ce paradigme apporte une réponse à la question actuelle de Françoise Héritier qui se propose de penser la différence sans hiérarchie.

Quelle est la réponse analytique à cette question ? La deuxième partie sur «La théorie psychanalytique de l'Éros » se charge de développer cetteérotologie freudienne. Moustapha Safouan a raison de rappeler avec force que la pulsion n'a pas de lien naturel au sexe de l'objet. Elle affiche une indépendance radicale à l'objet. Dès Les trois essais, la pulsion vient de l'objet perdu que Freud identifie à une perte corporelle vécue sur le plan narcissique. Le vrai problème surgit avec la phase phallique où il n'y $a$ pas de dommage corporel, ni de blessure narcissique. Moustapha Safouan nous aide à comprendre que la phase phallique est contemporaine de l'CEdipe et quec'est par la castration que le désirant s'allège du narcissisme en reconnaissant la dette symbolique au sein de son être. À cet effet, il nous donne sa lecture des fins d'analyses menées par Freud et leurs échecs. On lira que le désir du pénis préserve un manque qui ne se ravale pas au pénis et que la passivité masculine préserve un manque au-delà du don. C'est ce manque, creusé par la castration, quifait l'essence de la subjectivité et qui trouve à se symboliser par le phallus. Il reste maintenant à revisiter la sexualité freudienne avec ce concept du phallus.

On peut détruire une ville, mais pas son nom, ce qui prouve, selon Frege, qu'il y a de l'être dans le nom indépendam- ment de son existence. Cet exemple est le bienvenu pour introduire le lecteur au père symbolique dont le seul nom est un rempart, comme le disait Corneille à propos du Cid. On tient là un bel exemple d'un nom où se signifie une limite. C'est de ce nom que son porteur tire son autorité, tout comme le père. Ainsi, parler de la « Loi du père » ne fait qu'introduire la confusion entre sa fonction normativante et la potestas de la famille patriarcale appuyée sur la domination masculine. C'est là que Moustapha Safouan introduit la métaphore paternelle de Lacan, qui vient remanier le complexe d'đEdipe en déplaçant son centre vers la fonction phallique. La métaphorisation du phallus par le nom du père fait de ce dernier son équivalent de l'exclusion d'une jouissance souveraine et le pénis y trouve sa valeur. En somme, Moustapha Safouan nous rappelle combien cette relecture lacanienne de l'CEdipe se centre autour de la fonction phallique (ou de la castration) qui détermine le désir, par le manque qui engage l'être et l'avoir. Reste à savoir comment le sujet réalise ainsi son désir comme hétéro ou homo indépendamment de sonsexe.

Concernant «le désir masculin », Moustapha Safouan s'engage dans une argumentation des « mathèmes de la sexuation » de Lacan, en serrant au plus près la question de l'exception sur laquelle repose l'universel de la castration, sans pour autant impliquer un souverain. Ce que seule la psychanalyse est capable de penser, comme il le montre à partir de Carl Schmitt et de Hans Kelsen. " La principale découverte de la psychanalyse » sur l'identification sexuelle de l'homme concerne « la castration symbolique » (le manque insoluble dans le narcissisme) sans laquelle il n'aura aucune chance de faire l'amour, ce qu'exprime bien la formulation de Lacan : «II n'est passans 
l'avoir ». Pour Lacan, nous indique Moustapha Safouan, Freud a forgé son mythe de Totem et Tabou pour répondre à cet universel de la castration qui se fonde de l'exception. Bref, Lacan laisse tomber le « reflet patriarcal » de ce mythe pour un abord logique de la castration. La sexuation masculine passe par l'identification à l'exception du père qui jouit de toutes les femmes pour finalement s'inclure dans l'ensemble de tous les hommes soumis à la fonction phallique.

Dans le chapitre suivant sur « Le désir au féminin ", Moustapha Safouan présente la logique des mathèmes dans leur opposition à la logique classique, celle de l'Un, où chaque $x$ est un un, et le tout un un supérieur. À l'inverse, la logique de Lacan sur le modèle de la linguistique de F. de Saussure, s'appuie sur la pure différence sans former une totalité. Il en tire la conséquence que «la négation de l'existence de l'exception ou de l'exclusion, qui s'articule dans le premier mathème de la sexuation féminine, entraîne la négation de ce «tout». Nous obtenons ainsi le second mathème : pas-tout $\mathrm{x}$ est soumis à la fonction de la castration ". Comment comprendre ce pas-tout ? Moustapha Safouan soutient son explication de deux arguments qui lui permettent de conclure que la femme est prise de façon contingente dans la fonction phallique, à savoir que "le langage fait de la femme l'hétéros par excellence », et que " la castration ne s'applique pas positivement à son corps ". Reste la question de l'existence d'une jouissance féminine autre que celle de la jouissance phallique, où elle prend sa part. La démonstration de Moustapha Safouan reconnaît à la femme du fait de son rapport à la créativité la possibilité d'une jouissance « supplémentaire » à celle de la finitude de lajouissance phallique. Ce qui est dans le fil de son développement précédent sur le désir féminin.
Dans la troisième partie sur « La saga lacanienne », Moustapha Safouan présente Lacan comme le troisième « dissident » de l'IPA, après O. Rank et S. Ferenczi. Le fil rouge de ce livre est tenu par l'aiguille qui relie les trois parties en nouant la scientificité de la psychanalyse à la formation des analystes. L'hermétisme de l'IPA sur la didactique est, de ce point de vue, inacceptable. Le remède que Lacan y a apporté dans son École par l'invention de la passe, une procédure qui visait à recueillir un savoir sur le passage de l'analysant à l'analyste, s'est soldé selon ses propres mots par un « échec ». Moustapha Safouan analyse cet échec comme celui « de l'idéal d'une transmission scientifiquement assurée grâce à la psychanalyse pure (la didactique) ". Ainsi, le désir de l'analyste à l'œuvre dans la pratique analytique n'est pas du ressort des raisons, c'est-à-dire des fantasmes qui sous-tendent le désir de devenir analyste. Sans l'analyse de ces raisons, il n'y aurait pas d'analyste possible, mais le désir de l'analyste qui peut se signifier de façon fortuite ne se laisse pas prendre dans " un piège épistémologique ». Le désir de l'analyste est de l'ordre de la vérité. La passe a déçu l'attente d'un savoir qui reposait "sur l'assimilation de la conduite de l'analyse didactique à une opération scientifique ». En somme, on ne peut pas « se servir de l'éclair comme d'une lampe ».

Dans sa «Conclusion » Moustapha Safouan décrit l'analyse comme la pratique d'un « art » qui ne s'apprend pas parce qu'il dépend de la sensibilité du praticien aux effets constituants de la parole. La formation qu'il dit devoir à Lacan est liée à sa position de venir soutenir ses efforts pour « inventer la psychanalyse » à son tour.

Nous avons aujourd'hui sous nos yeux un travail considérable de réin- 
vention de la psychanalyse par un analyste qui a fait partie des premiers élèves de Lacan et qui nous fait revivre « les années Lacan », sans jamais en rien céder sur son projet scientifique, son désir pour la psychanalyse. Il réalise par cette œuvre le projet de Lacan qu'il a prononcé lors du dernier congrès de son École : " chaque analyste réinvente la façon dont la psychanalyse peut durer ». J'insiste sur les mots que j'ai mis en italiques, la durée de la psychanalyse dépendra, comme le dit Moustapha Safouan, de « sa capacité à contribuer à l'intelligence de notre époque, et aux métamorphoses de l'Éros, autrement qu'en poussant des cris d'alarme ».

Ce livre ouvre une nouvelle ère dans la psychanalyse par l'examen qu'il fait du projet scientifique de la psychanalyse, de ses avancées et de ses stagnations, au regard de son institutionnalisation, des chefs et des servitudes que l'institution appelle et ceci sans complaisance envers les maîtres fondateurs et leurs « héritiers ».

La psychanalyse y gagne et le débat s'ouvre. 